\title{
Differences in the importance of microcephaly, dysmorphism, and epilepsy in the detection of pathogenic CNVs in ID and ASD patients
}

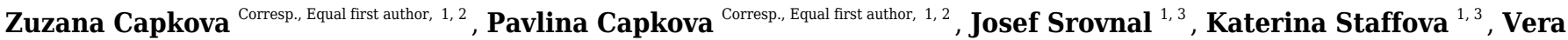 \\ Becvarova $^{4}$, Marie Trkova ${ }^{4}$, Katerina Adamova ${ }^{1,2}$, Alena Santava ${ }^{1,2}$, Vaclava Curtisova $^{1,2}$, Marian Hajduch ${ }^{3}$, \\ Martin Prochazka ${ }^{1,2}$ \\ 1 Department of Medical Genetics, University Hospital Olomouc, Olomouc, Czech Republic \\ 2 Department of Medical Genetics / Faculty of Medicine and Dentistry, Palacky University Olomouc, Olomouc, Czech Republic \\ 3 Institute of Molecular and Translational Medicine / Faculty of Medicine and Dentistry, Palacky University Olomouc, Olomouc, Czech Republic \\ 4 Gennet, s.r.o., Prague, Czech Republic
}

Corresponding Authors: Zuzana Capkova, Pavlina Capkova

Email address: zuzana.capkova@fnol.cz, Pavlina.Capkova@fnol.cz

Background. Autism spectrum disorders (ASD) and intellectual disabilities (ID) are heterogeneous and complex developmental diseases with significant genetic backgrounds and overlaps of genetic susceptibility loci. Copy number variants (CNVs) are known to be frequent causes of these impairments. However, the clinical heterogeneity of both disorders causes the diagnostic efficacy of CNV analysis to be modest. This could be resolved by stratifying patients according to their clinical features.

Aim. First, we sought to assess the significance of particular clinical features for the detection of pathogenic CNVs in separate groups of ID and ASD patients and determine whether and how these groups differ from each other in the significance of these variables. Second, we aimed to create a statistical model showing how particular clinical features affect the probability of pathogenic CNV findings.

Method. We tested a cohort of 204 patients with ID ( $N=90)$ and ASD ( $N=114)$ for the presence of pathogenic CNVs. We stratified both groups according to their clinical features. Fisher's exact test was used to determine the significance of these variables for pathogenic CNV findings. Logistic regression was used to create a statistical model of pathogenic CNV findings.

Results. The frequency of pathogenic CNV was significantly higher in the ID group than in the ASD group - $18(19.78 \%)$ versus $8(7 \%)(p<0.004)$. Microcephaly showed a significant association with pathogenic findings in ID patients $(p<0.01)$ according to Fisher's exact test, whereas epilepsy showed a significant association with pathogenic findings in ASD patients $(p<0.01)$. The probability of pathogenic CNV findings when epilepsy occurred in ASD patients was more than two times higher than if epilepsy cooccurred with ID (29.6\%/14.0\%). Facial dysmorphism was a significant variable for detecting pathogenic CNVs in both groups (ID $p=0.05$, ASD $p=0.01$ ). However, dysmorphism increased the probability of pathogenic CNV detection in the ID group nearly twofold compared to the ASD group (44.4\%/23.7\%). The presence of macrocephaly in the ASD group showed a $25 \%$ probability of pathogenic CNV findings by logistic regression, but this was insignificant according to Fisher's exact test. The probability of detecting pathogenic CNVs decreases up to $1 \%$ in the absence of dysmorphism, macrocephaly, and epilepsy in the ASD group. 
Conclusion. Dysmorphism, microcephaly, and epilepsy increase the probability of pathogenic CNV findings in ID and ASD patients. The significance of each feature as a predictor for pathogenic CNV detection differs depending on whether the patient has only ASD or ID. The probability of pathogenic CNV findings without dysmorphism, macrocephaly, or epilepsy in ASD patients is low. Therefore theefficacy of CNV analysis is limited in these patients. 
1 Differences in the importance of microcephaly, dysmorphism, and epilepsy in the detection 2 of pathogenic CNVs in ID and ASD patients

3

4 Zuzana Capkova ${ }^{1,2}$, Pavlina Capkova ${ }^{1,2}$, Josef Srovnal ${ }^{1,3}$, Katerina Staffova ${ }^{3}$, Vera

5 Becvarova $^{4}$, Marie Trkova ${ }^{4}$, Katerina Adamova ${ }^{1,2}$, Alena Santava ${ }^{1,2}$, Vaclava Curtisova ${ }^{1,2}$,

6 Marian Hajduch $^{3}$, Martin Prochazka ${ }^{1,2}$

7

8

$9{ }^{1}$ Department of Medical Genetics, University Hospital Olomouc, Olomouc, Czech Republic.

${ }^{2}$ Department of Medical Genetics, Faculty of Medicine and Dentistry, Palacky University

11 Olomouc, Olomouc, Czech Republic.

${ }^{3}$ Institute of Molecular and Translational Medicine, Faculty of Medicine and Dentistry, Palacky University Olomouc, Olomouc, Czech Republic.

${ }^{4}$ Gennet, s.r.o., Prague, Czech Republic

15

Corresponding Authors:

I.P. Pavlova 6, Olomouc, 77900, Czech Republic

Email address: Zuzana.Capkova@fnol.cz

Pavlína Čapková ${ }^{1,2}$

I.P. Pavlova 6, Olomouc, 77900, Czech Republic 
32 Email address: Pavlina.Capkova@fnol.cz

33

34

Peer) reviewing PDF | (2019:05:37470:1:2:NEW 24 Sep 2019) 
Background. Autism spectrum disorders (ASD) and intellectual disabilities (ID) are heterogeneous and complex developmental diseases with significant genetic backgrounds and overlaps of genetic susceptibility loci. Copy number variants (CNVs) are known to be frequent causes of these impairments. However, the clinical heterogeneity of both disorders causes the diagnostic efficacy of CNV analysis to be modest. This could be resolved by stratifying patients according to their clinical features.

Aim. First, we sought to assess the significance of particular clinical features for the detection of pathogenic CNVs in separate groups of ID and ASD patients and determine whether and how these groups differ from each other in the significance of these variables. Second, we aimed to create a statistical model showing how particular clinical features affect the probability of pathogenic $\mathrm{CNV}$ findings.

Method. We tested a cohort of 204 patients with ID $(\mathrm{N}=90)$ and ASD $(\mathrm{N}=114)$ for the presence of pathogenic CNVs. We stratified both groups according to their clinical features. Fisher's exact test was used to determine the significance of these variables for pathogenic CNV findings. Logistic regression was used to create a statistical model of pathogenic CNV findings.

Results. The frequency of pathogenic CNV was significantly higher in the ID group than in the ASD group - $18(19.78 \%)$ versus $8(7 \%)(p<0.004)$. Microcephaly showed a significant association with pathogenic findings in ID patients $(p<0.01)$ according to Fisher's exact test, whereas epilepsy showed a significant association with pathogenic findings in ASD patients $(p<$ 0.01). The probability of pathogenic CNV findings when epilepsy occurred in ASD patients was more than two times higher than if epilepsy co-occurred with ID $(29.6 \% / 14.0 \%)$. Facial dysmorphism was a significant variable for detecting pathogenic CNVs in both groups (ID $p=$ 0.05 , ASD $p=0.01)$. However, dysmorphism increased the probability of pathogenic CNV detection in the ID group nearly twofold compared to the ASD group (44.4\%/23.7\%). The presence of macrocephaly in the ASD group showed a $25 \%$ probability of pathogenic CNV findings by logistic regression, but this was insignificant according to Fisher's exact test. The probability of detecting pathogenic CNVs decreases up to $1 \%$ in the absence of dysmorphism, macrocephaly, and epilepsy in the ASD group. 
63 Conclusion. Dysmorphism, microcephaly, and epilepsy increase the probability of pathogenic 64 CNV findings in ID and ASD patients. The significance of each feature as a predictor for pathogenic $\mathrm{CNV}$ detection differs depending on whether the patient has only ASD or ID. The probability of pathogenic CNV findings without dysmorphism, macrocephaly, or epilepsy in ASD

67 patients is low. Therefore the efficacy of CNV analysis is limited in these patients.

68

69

Intellectual disabilities (ID) and autism spectrum disorders (ASD) are relatively common and their impacts on patients, their families, and society are well known (Tonnsen et al., 2016; Matson \& Shoemaker, 2009; Schaefer, 2016). Autism spectrum disorders involve a broad range of conditions characterised by deficits in social skills, repetitive behaviours, impaired speech, and nonverbal communication (Bourgeron, 2016; Schaefer, 2016). Intellectual disabilities involve problems with general mental abilities, which affect intellectual functioning and adaptive functioning (Quintela et al., 2017).

The rates of ID and ASD are approximately 3 in 100 and 1 in 68, respectively, in the worldwide child population (Harripaul et al., 2017). Despite the fact that these conditions are distinct entities, they share some characteristics: a preponderance of affected males (Lai et al., 2015; Mitra et al., 2016; Jacquemont et al., 2014), a genetic background (Diaz-Beltran et al., 2017; Gonzalez-Mantilla et al., 2016), and accompaniment by clinical features such as hyperactivity (ADHD), epilepsy, speech impairment, and learning disabilities. In both disorders, skeletal abnormalities of the skull—microcephaly, macrocephaly, dysmorphic features — are frequently described (Viñas-Jornet et al., 2018; Matson \& Shoemaker, 2009). Multiple studies have demonstrated the genetic bases of ASD and ID, including chromosome abnormalities, single genes, copy number variants (CNVs), and multifactorial inheritance (Krishnan et al., 2016; de la 
86 Torre-Ubieta et al., 2016; Egger et al., 2014). Despite all efforts, the aetiology of these

87 conditions is still not fully understood (Stessman et al., 2017).

88 Portfolio methods commonly used to find the causes of ASD and ID involve conventional

89 karyotyping, fluorescent in situ hybridisation (FISH), multiplex ligation-probe dependent

90 amplification (MLPA), and chromosomal microarrays (CMA) - recently accepted as a frontline

91 method (Battaglia et al., 2013) - which have resulted in the discovery of many genetic variants

92 (de la Torre-Ubieta et al., 2016; Schaefer, 2016; Hehir-Kwa et al., 2013; Merikangas et al., 93 2015).

94 The overlap of genetic susceptibility loci has been described previously in diagnoses of both

95 disorders (Matson \& Shoemaker, 2009; Lowther et al., 2017) together with other

96 neuropsychiatric disorders such as schizophrenia, Alzheimer's disease, Parkinson's disease, and

97 others. (Wolfe et al., 2017; Sokol et al., 2011; Catalá-López et al., 2014). It is assumed that the

98 incomplete penetrance and variable expression of CNVs are the causes of different clinical

99 phenotypes of the same CNV (Lowther et al., 2017). This situation usually causes patients with

100 ASD and ID to be grouped together based on overlapping clinical and genetic features, and so

101 the efficacy of genetics-based diagnostic methods is relatively low (Xu et al., 2018; Peycheva et

102 al., 2018, Schaefer \& Mendelsohn, 2013, Chan et al. 2018). On the other hand, the yields of

103 these tests are strongly influenced by the clinical features that accompany ID and ASD (Ho et al.,

104 2016, Miller et al., 2010; Beaudet, 2013, Jacquemont et al., 2006).

105 We assessed the impact of particular clinical features (ADHD, epilepsy, growth defects,

106 congenital malformations, microcephaly, macrocephaly, and dysmorphism) on the detection of

107 pathogenic CNVs in separate ID and ASD groups and determined whether and how these groups 108 differ from each other. 


\section{Materials}

110 A total of 204 patients of Caucasian descent with intellectual disabilities (ID) and autism

111 spectrum disorders (ASD) referred to genetic counselling were enrolled in this retrospective

112 study. All patients underwent rigorous examinations by paediatricians, neurologists,

113 psychiatrists, and geneticists, including metabolic tests and brain imaging. Metabolic disorders

114 were excluded by the Department of Clinical Biochemistry, Palacky University Olomouc, Czech

115 Republic, through biochemical screening. Peripheral blood samples were collected after genetic

116 counselling in the Department of Medical Genetics at the University Hospital Olomouc, Czech

117 Republic, during the years 2012-2018. Part of this counselling was the collection of informed

118 consent from parents or guardians of the patients in accordance with the Declaration of Helsinki.

119 The Institutional Review Board of the University Hospital and the Faculty of Medicine and

120 Dentistry, Palacky University Olomouc, granted a permit for this study (IRB number 96/17). The

121 patients were stratified into two groups - 90 patients with solely intellectual disabilities (ID) (51

122 male, 39 female) aged 5-35 years old, and 114 patients with autistic spectrum disorders (ASD)

123 (78 male, 36 female) aged 3-18 years old. The ASD cohort involved patients with $(\mathrm{N}=96)$ and

124 without $(\mathrm{N}=18)$ intellectual impairment, but they differ from the ID group in that they have

125 been diagnosed with autism, which was taken as the primary reason for the investigation. ASD

126 individuals were diagnosed with ASD by clinicians after performing the Autism Diagnosis

127 Observation Schedule. Subjects with pervasive developmental disorders and varying levels of

128 impairment were diagnosed with broad-spectrum disorder, which involves conditions such as

129 pervasive developmental disorders not otherwise specified (PDD-NOS) and Asperger's

130 syndrome.

131 Methods

Peer) reviewing PDF | (2019:05:37470:1:2:NEW 24 Sep 2019) 
132 The study design was retrospective. Patients' clinical data were collected from their most up-to-

133 date medical records to eliminate changes in the definitions of ID and ASD and to reflect the

134 actual diagnosis of each patient at that period of the time. General observations of the clinical

135 features in patients were made by genetic counsellors or specialists. Scored features were

136 ADHD; epilepsy; microcephaly (head circumference $<2$ nd percentile); macrocephaly (head

137 circumference $>$ 98th percentile); facial dysmorphism (abnormalities of the eye slits, superciliary

138 arches, nose, lips, philtrum, ears, jaws, palatum durum, face shape, and

139 hairline); developmental defects of the heart, urogenital system, and brain; and growth

140 restrictions.

141 Patients with chromosomal aberrations and FMR1 mutations were excluded from the study.

142 DNA from peripheral blood isolated by the saline method was used for CNV analysis by MLPA

143 and/or CMA.

144 MLPA tests were performed with SALSA ${ }^{\circledR}$ MLPA ${ }^{\circledR}$ probes for testing subtelomeric regions 145 (P070 Subtelomeres Mix 2B, P036 Subtelomeres Mix 1), the most frequent microdeletion or 146 microduplication syndromes (P245 Microdeletion Syndromes-1A, P297 Microdeletion

147 Syndromes-2), autistic and X-linked intellectual disability susceptibility regions (P343 Autism-1, 148 P106 MRX). MLPA analysis adhered the protocol recommended by the manufacturer

149 (www.mlpa.com, 31 August 2018). Capillary electrophoresis (CE) was used for the

150 determination of PCR products using an ABI 3130 genetic analyser provided by the Gene

151 Mapper software (Applied Biosystems, Foster City, CA, USA). The Coffalyser program was

152 used for CNV calling (MRC-Holland, Amsterdam, the Netherlands).

153 Cytoscan HD (Affymetrix, Santa Clara, CA, USA) and CytoSNP-12 (Illumina, San Diego, CA,

154 USA) instruments were used for CMA analysis according to the manufacturers' protocols 
155 (www.affymetrix.com, www.illumina.com, 31 August 2018). The data discussed in this

156 publication have been deposited in NCBI's Gene Expression Omnibus database (Edgar,

157 Domrachev \& Lash, 2002) and are accessible using GEO Series accession number GSE132453.

158 The programs CHAS v1.2.2 (Affymetrix, Santa Clara, CA, USA) and Illumina KaryoStudio 1.3

159 (Genome Studio v2011.1) were used for CNV calling. Pathogenic CNVs were determined using

160 curated databases (ISCA, Decipher, SFARI, DGV) and with the acceptance of guidelines

161 (Kearney et al., 2011, Schaefer \& Mendelsohn, 2013).

162 The significance of particular clinical features for the detection of pathogenic CNVs was

163 determined by Fisher's exact test. A statistical model was prepared using a forward/stepwise

164 logistic regression model to resolve how particular features affect the probability of pathogenic

165 CNV findings in both groups. Both tests were performed in each group (ID and ASD) by an

166 analytical company (ACREA, Prague, Czech Republic).

\section{Results}

168 Dysmorphism, microcephaly, and developmental defects (heart, urogenital system, and brain)

169 were significantly more abundant in patients with solely ID $(\mathrm{p}<0.5)$, whereas ADHD was more

170 prevalent in the ASD group $(\mathrm{p}<0.5)$ (Table 1).

171 We detected pathogenic CNVs in 26 patients (12.7\%); altogether: ID = $18(19.8 \%) ; \mathrm{ASD}=8$

$172(7.0 \%)$. The frequency of pathogenic CNVs was significantly higher in the ID group than in the

173 ASD group $(\mathrm{p}<0.004)$. This $\mathrm{p}$-value decreased when 18 ASD patients without intellectual

174 disabilities were excluded $(p=0.02)$. The frequency of pathogenic CNVs in both groups (ID and 175 ASD) taken together and in each group separately and stratified according to discrete features are

176 shown in Table 2 and Figures 1, 2, and 3. Patients with pathogenic CNVs are described in

177 Supplement Table 1. 
178 Fisher's exact test of the groups separated according to ID or ASD showed that microcephaly ( $p$

$179=0.01)$ in the ID group and epilepsy $(\mathrm{p}=0.01)$ in the ASD group were significant for the finding

180 of CNVs, whereas dysmorphism is significant in both groups (ID $p=0.05$, ASD $p=0.01$ ). ASD

181 patients with macrocephaly and ADHD expressed a higher percentage of pathogenic variants,

182 though this was not as statistically significant as some growth defects in the ID group (p=ns).

183 Developmental defects of the heart, urogenital system, and brain did not achieve statistical

184 significance in either group $(\mathrm{p}=\mathrm{ns})$. The summary in Table 3 shows the achieved significance of

185 particular clinical features to pathogenic CNV findings in the ASD and ID groups using Fisher's

186 exact test.

187 We created a statistical model and calculated the probability of pathogenic CNV findings in ASD

188 and ID patients with each significant feature from Fisher's exact test. Macrocephaly in ASD

189 patients was added to the model based on the close percentage of pathogenic CNVs

190 to dysmorphism. In the ID group, microcephaly was significant only when separate and fell out

191 of the overall model. Dysmorphism achieved a 44.4\% probability of pathogenic CNV findings in

192 the ID group, in contrast with the ASD group, which reached only $23.7 \%$. These differences

193 were nearly twice as high for the ID group compared to the ASD group $(\mathrm{p}=0.052)$.

194 The risk of pathogenic CNVs in patients with epilepsy was $29.6 \%$ in the ASD group and $14 \%$ in

195 the ID group, meaning the risk was more than twice as high for the ASD group than for the ID

196 group $(\mathrm{p}=0.003)$. This difference was significant and it implies that for the detection of

197 pathogenic CNVs in patients with epilepsy it is important to know whether this patient is

198 classified as ID or ASD (Figure 4).

199 Added macrocephaly showed a 25\% pathogenic CNV probability in ASD patients, which is

200 higher than dysmorphism (23.7\%). 
201 The absence of dysmorphism, macrocephaly, and epilepsy decreases the probability of detecting

202 pathogenic CNVs to $1 \%$ in the ASD group (Figures 5). The calculation of logistic regression and

203 formulas for the calculation of pathogenic CNV probability in the ASD group are shown in

204 Supplement Table 2 and Figure 6. No pathogenic CNVs were found in autistic patients without

205 intellectual disability. Apart from one case of epilepsy, no microcephaly, macrocephaly,

206 or dysmorphism were found in this subgroup, nor were any pathogenic CNVs. Although it would

207 be interesting to compare this subgroup with the ID cohort, this could not be accurately

208 calculated due to the cohort size $(\mathrm{N}=18)$.

209 Discussion

210 Autism spectrum disorders (ASD) and intellectual disabilities (ID) are overlapping genetically

211 conditioned developmental diseases that are frequently accompanied by ADHD, epilepsy,

212 microcephaly, macrocephaly, dysmorphism, developmental defects, and/or growth defects

213 (Whittington et al., 2018; Li et al., 2018; Mulle et al., 2014, Bourgeron, 2016; Schaefer, 2016;

214 Quintela et al., 2017). Copy number variants (CNVs) have been identified as one of the possible

215 cause of these diseases (Miller et al., 2010, Battaglia et al., 2013, de la Torre-Ubieta et al., 2016;

216 Schaefer, 2016; Hehir-Kwa et al., 2013; Merikangas et al., 2015). CNV analysis is recommended

217 in patients with ASD and/or ID as a frontline test, and the efficacy of this approach is $8.7 \%$ -

218 14.7\% in patients with ID and 12\% in patients with ASD (Xu et al., 2018; Peycheva et al., 2018,

219 Miller et al., 2010). The yields of CNV analyses are influenced by the accompanying clinical

220 features in ID/ASD patients (Ho et al., 2016).

221 Pathogenic CNVs have been detected in higher percentages (15\%-20\%) in ID and ASD cohorts

222 when additional clinical features (e.g. micro-/macrocephaly, dysmorphism, developmental and

223 growth defects) are present (Miller et al., 2010; Beaudet, 2013, Jacquemont et al., 2006). In some 
224 studies, dysmorphia and/or microcephaly increased the percentage of pathogenic CNVs up to $22545.8 \%$ in ID and ASD cohorts (Jacquemont et al., 2006, Chan et al. 2018, Miles et al. 2011). In 226 published results, patients with ASD and ID are usually grouped based on overlapping clinical 227 and genetic features, but we were interested in the impact of each clinical feature on each group 228 separately (ID, ASD) (Miller et al., 2010; Beaudet, 2013, Jacquemont et al., 2006, Chan et al. 229 2018).

230 We are able to confirm that dysmorphism increases the probability of pathogenic

231 CNV detection in ID and ASD patients (Miller et al., 2010; Beaudet, 2013, Jacquemont et al., 232 2006). The calculated probability of pathogenic CNVs in patients with dysmorphism was $44.4 \%$ and $23.7 \%$ in the ID cohort and ASD cohort, respectively, meaning the risk was nearly twice as

234 high for the ID group than for the ASD group $(\mathrm{p}=0.052)$. Dysmorphic features have proven to 235 be the most prominent predictor for pathogenic CNVs in the ID group.

236 Defects of the skull, namely size, are important clinical features for findings of pathogenic CNVs 237 in ID and/or ASD patients (Qiao, 2009; Hultman et al., 2010; Shaw-Smith et al., 2004;

238 Bernardini et al., 2010, Blanken et al., 2018; Klein, Sharifi-Hannauer \& Martinez-Agosto, 2013).

239 The co-segregation of microcephaly and dysmorphism has frequently been associated with the

240 presence of pathogenic CNVs in groups of ID children (Qiao, 2009; Hultman et al., 2010; Shaw-

241 Smith et al., 2004; Bernardini et al., 2010), whereas macrocephaly might be associated with

242 certain subtypes of autism (Blanken et al., 2018; Klein, Sharifi-Hannauer \& Martinez-Agosto, 243 2013). Our study confirmed that microcephaly is more frequently found in ID patients than in 244 ASD patients and that the probability of finding pathogenic CNVs is significantly higher in the 245 ID group than in the ASD group. 
246 We cannot prove that macrocephaly is significantly associated with pathogenic CNV findings in

247 both groups (ID $p=0.338$; ASD $p=0.256$ ). Even though the difference was not significant,

248 macrocephaly was included in the statistical model of logistic regression in the ASD group in

249 order to situate our work in relation to previously published works (Blanken et al., 2018; Klein,

250 Sharifi-Hannauer \& Martinez-Agosto, 2013). Macrocephaly increased the probability of

251 pathogenic CNV detection in affected ASD children up to $25 \%$, more

252 than dysmorphism (23.7\%). The explanation for this is that isolated macrocephaly has low

253 predictive value, but in combination with other traits it could profoundly increase the probability

254 of pathogenic CNV detection.

255 There are still controversies over the association between autism and epilepsy (Amiet et al.,

256 2013; Lee, Smith \& Paciorkowski, 2015; Berg \& Plioply, 2012). A recent population-based

257 study found that $44 \%$ of children with ASD received a subsequent diagnosis of epilepsy, and

$25854 \%$ of children with epilepsy received a subsequent diagnosis of ASD (Jokiranta et al., 2014).

259 The role of pathogenic CNVs in epilepsy has been previously described (Olson et al., 2014,

260 Viscidi et al., 2013). We proved that epilepsy is significant for pathogenic CNV findings in ASD

261 patients but was insignificant in patients with ID, even though the proportion of patients with

262 epilepsy was the same in both groups.

263 ASD and ID are frequently accompanied by ADHD, developmental and growth defects,

264 epilepsy, micro-/macrocephaly, and/or dysmorphism (Whittington et al., 2018; Li et al., 2018;

265 Mulle et al., 2014, Bourgeron, 2016; Schaefer, 2016; Quintela et al., 2017).

266 ADHD manifested more frequently in the ASD cohort than in the ID cohort. However, ADHD,

267 similar to developmental (heart, urogenital system, and brain) and growth defects, is a variable

268 that appeared to be insignificant in the detection of pathogenic CNVs in both groups in our work. 
269 We assume that causes other than pathogenic CNVs underlie ADHD (Kim et al. 2017).

270 Interestingly, developmental (heart, urogenital system, and brain) and growth defects are part of

271 some syndromes that accompany ASD and/or ID, but both were insignificant in their effects on

272 findings of pathogenic CNVs in our patients (Whittington et al., 2018; Li et al., 2018; Mulle et

273 al., 2014, Bourgeron, 2016; Schaefer, 2016; Quintela et al., 2017).

274 Finally, Ho et al. (2016) tested more than 10,000 patients with neurodevelopmental diseases,

275 namely developmental delays / intellectual disabilities and autism spectrum disorders, and noted

276 that the percentage of pathogenic CNVs in patients with neurodevelopmental disorders increases

277 when autistic patients are excluded (28.1\% vs 33.0\%). This supports our results. The probability

278 of pathogenic CNVs was calculated to be $1 \%$ for ASD patients without dysmorphism, epilepsy,

279 or macrocephaly. The result implies that the relevance of CNV analysis is significantly

280 influenced by accompanying features, especially in ASD patients. This fact generally goes

281 against the accepted recommendation of CNV analysis in patients with ASD but without

282 additional clinical features (Ho et al., 2016).

283 We confirmed the impact of dysmorphic features and microcephaly and added epilepsy as a

284 predictor for the finding of pathogenic CNVs and highlighted the differing impacts of each

285 clinical feature on each group (ID, ASD) in searching for pathogenic CNVs. In addition, we

286 identified low rates of pathogenic variants in patients with ASD but without intellectual

287 disability, dysmorphism, macrocephaly, or epilepsy. However, our results did not asses the

288 influence of other variables that could affect CNV rates (e.g. gender).

289 Conclusion: 
290 The presences of dysmorphism, microcephaly, and epilepsy increased the detection rate of

291 pathogenic CNVs in patients with ID and ASD in our study, but the significance of each feature

292 is different for each group. Microcephaly is a significant predictor for the risk of pathogenic

293 CNVs in patients with intellectual disabilities but not in patients with autism spectrum disorders,

294 whereas epilepsy is a significant predictor for the risk of pathogenic CNVs in patients with ASD

295 but not in patients with ID. Dysmorphism is a significant predictor in both groups, but is nearly

296 twice as high in patients with ID than patients with ASD. Macrocephaly may increase the

297 probability of pathogenic CNV findings in ASD patients. ID and ASD patients with ADHD or

298 developmental and growth defects as well as some ASD patients without dysmorphism, epilepsy,

299 or macrocephaly have a very low probabilities of pathogenic CNVs, which is contrary to the

300 generally accepted recommendation of CNV analysis in patients with ID and ASD.

301 References

302

303

304

305

306

307

308

309
Amiet C., Gourfinkel-An I., Laurent C., Bodeau N., Génin B., Leguern E., Tordjman S., Cohen

D. 2013. Does epilepsy in multiplex autism pedigrees define a different subgroup in terms of

clinical characteristics and genetic risk?. Molecular Autism. DOI: 10.1186/2040-2392-4-47.

Battaglia A., Doccini V., Bernardini L., Novelli A., Loddo S., Capalbo A., Filippi T., Carey J.C.

2013. Confirmation of chromosomal microarray as a first-tier clinical diagnostic test for

individuals with developmental delay, intellectual disability, autism spectrum disorders and

dysmorphic features. European Journal of Paediatric Neurology. DOI:

10.1016/j.ejpn.2013.04.010.

Peer) reviewing PDF | (2019:05:37470:1:2:NEW 24 Sep 2019) 
310 Bernardini L., Alesi V., Loddo S., Novelli A., Bottillo I., Battaglia A., Digilio M.C., Zampino

311 G., Ertel A., Fortina P., Surrey S., Dallapiccola B. 2010. High-resolution SNP arrays in mental

312 retardation diagnostics: how much do we gain?. European Journal of Human Genetics. DOI:

313 10.1038/ejhg.2009.154.

314 Blanken L.M.E., Dass A., Alvares G., van der Ende J., Schoemaker N.K., El Marroun H., Hickey

315 M., Pennell C., White S., Maybery M.T., Dissanayake C., Jaddoe V.W.V., Verhulst F.C.,

316 Tiemeier H., McIntosh W., White T., Whitehouse A. 2018. A prospective study of fetal head

317 growth, autistic traits and autism spectrum disorder. Autism Research. DOI: 10.1002/aur.1921.

318 Bourgeron T. 2016. Current knowledge on the genetics of autism and propositions for future

319 research. Comptes Rendus Biologies. DOI: 10.1016/j.crvi.2016.05.004.

320 Beaudet A.L. 2013. The Utility of Chromosomal Microarray Analysis in Developmental and

321 Behavioral Pediatrics. Child Development. DOI: 10.1111/cdev.12050.

322 Berg A.T., Plioplys S. 2012. Epilepsy and autism: is there a special relationship?. Epilepsy

323 Behav. DOI: 10.1016/j.yebeh.2012.01.015.

324 Catalá-López F., Suárez-Pinilla M., Suárez-Pinilla P., Valderas J.M., Gómez-Beneyto M.,

325 Martinez S., Balanzá-Martínez V., Climent J., Valencia A., McGrath J., Crespo-Facorro B.,

326 Sanchez-Moreno J., Vieta E., Tabarés-Seisdedos R. 2014. Inverse and Direct Cancer 
327 Comorbidity in People with Central Nervous System Disorders: A Meta-Analysis of Cancer

328 Incidence in 577,013 Participants of 50 Observational Studies. Psychotherapy and

329 Psychosomatics. DOI: 10.1159/000356498.

330 Chan P.Y.T., Luk H.M., Lee F.M.Y., Lo I.F.M. 2018. Genetic profile and clinical application of

331 chromosomal microarray in children with intellectual disability in Hong Kong. Hong Kong

332 Medical Journal. DOI: 10.12809/hkmj187260.

333 Diaz-Beltran L., Esteban F.J., Varma M., Ortuzk A., David M., Wall D.P. 2017. Cross-disorder

334 comparative analysis of comorbid conditions reveals novel autism candidate genes. BMC

335 Genomics DOI: 10.1186/s12864-017-3667-9.

336 Egger G., Roetzer K.M., Noor A., Lionel A.C., Mahmood H., Schwarzbraun T., Boright O.,

337 Mikhailov A., Marshall C.R., Windpassinger C., Petek E., Scherer S.W., Kaschnitz W., Vincent

338 J.B. 2014. Identification of risk genes for autism spectrum disorder through copy number

339 variation analysis in Austrian families. Neurogenetics. DOI: 10.1007/s10048-014-0394-0.

340 Gonzalez-Mantilla A.J., Moreno-De-Luca A., Ledbetter D.H., Martin C.L. 2016. A Cross-

341 Disorder Method to Identify Novel Candidate Genes for Developmental Brain Disorders. JAMA

342 Psychiatry. DOI: 10.1001/jamapsychiatry.2015.2692. 
343 Harripaul R., Noor A., Ayub M., Vincent J.B. 2017. The Use of Next-Generation Sequencing for

344 Research and Diagnostics for Intellectual Disability. Cold Spring Harbor Perspectives in

345 Medicine. DOI: 10.1101/cshperspect.a026864.

346 Hehir-Kwa J.Y., Pfundt R., Veltman J.A., de Leeuw N. 2013. Pathogenic or not? Assessing the

347 clinical relevance of copy number variants. Clinical Genetics. DOI: 10.1111/cge.12242.

348 Ho K.S., Twede H., Vanzo R., Harward E., Hensel C.H., Martin M.M., Page S., Peiffer A.,

349 Mowery-Rushton P., Serrano M., Wassman E.R. 2016. Clinical Performance of an Ultrahigh

350 Resolution Chromosomal Microarray Optimized for Neurodevelopmental Disorders. BioMed

351 Research International. DOI: 10.1155/2016/3284534.

352 Hultman C.M., Sandin S., Levine S.Z., Lichtenstein P., Reichenberg A. Advancing paternal age

353 and risk of autism: New evidence from a population-based study and a meta-analysis of

354 epidemiological studies. Molecular Psychiatry. DOI: 10.1038/mp.2010.121.

355 Quintela I., Eirís J., Gómez-Lado C., Pérez-Gay L., Dacruz D., Cruz R., Castro-Gago M.,

356 Míguez L., Carracedo Á., Barros F. 2017. Copy number variation analysis of patients with

357 intellectual disability from North-West Spain. Gene. DOI: 10.1016/j.gene.2017.05.032.

358 Jacquemont S., Coe B P., Hersch M., Duyzend M.H., Krumm N., Bergmann S., Beckmann J.S.,

359 Rosenfeld J.A., Eichler E.E. 2014. A Higher Mutational Burden in Females Supports a "Female 
360 Protective Model” in Neurodevelopmental Disorders. The American Journal of Human Genetics.

361 DOI: 10.1016/j.ajhg.2014.02.001.

362 Jokiranta E., Sourander A., Suominen A., Timonen-Soivio L., Brown A.S., Sillanpää M. 2014.

363 Epilepsy Among Children and Adolescents with Autism Spectrum Disorders: A Population-

364 Based Study. Journal of Autism and Developmental Disorders. DOI: 10.1007/s 10803-014-2126-

3656.

366 Kim D.S., Burt A.A., Ranchalis J.E., Wilmot B., Smith J.D., Patterson K.E., Coe B.P., Li Y.K.,

367 Bamshad M.J., Nikolas M., Eichler E.E., Swanson J.M., Nigg J.T., Nickerson D.A., Jarvik G.P.

368 2017. Sequencing of sporadic Attention-Deficit Hyperactivity Disorder (ADHD) identifies novel

369 and potentially pathogenic de novo variants and excludes overlap with genes associated with

370 autism spectrum disorder. Am J Med Genet B Neuropsychiatr Genet. DOI:

371 10.1002/ajmg.b.32527.

372 Klein S., Sharifi-Hannauer P., Martinez-Agosto J.A. 2013. Macrocephaly as a Clinical Indicator

373 of Genetic Subtypes in Autism. Autism Research. DOI: 10.1002/aur.1266.

374 Krishnan A., Zhang R., Yao V., Theesfeld C.L., Wong AK., Tadych A., Volfovsky N., Packer

375 A., Lash A., Troyanskaya O.G. 2016. Genome-wide prediction and functional characterization of

376 the genetic basis of autism spectrum disorder. Nature Neuroscience. DOI: $10.1038 / \mathrm{nn} .4353$. 
377 Lai M.C., Lombardo M.V., Auyeung B., Chakrabarti B., Baron-Cohen S. 2015. Sex/Gender

378 Differences and Autism: Setting the Scene for Future Research. Journal of the American

379 Academy of Child \& Adolescent Psychiatry. DOI: 10.1016/j.jaac.2014.10.003.

380 Lee B.H., Smith T., Paciorkowski A.R. 2015. Autism spectrum disorder and epilepsy: Disorders

381 with a shared biology. Epilepsy \& Behavior. DOI: 10.1016/j.yebeh.2015.03.017.

382 Li H., Du J., Li W., Cheng D., He W., Yi D., Xiong B., Yuan S., Tu C., Meng L., Luo A., Lin

383 G., Lu G., Tan Y.Q. 2018. Rare partial octosomy and hexasomy of 15q11-q13 associated with

384 intellectual impairment and development delay: report of two cases and review of

385 literature. Molecular Cytogenetics. DOI: 10.1186/s13039-018-0365-5.

386 Lowther C., Costain G., Baribeau D.A., Bassett AS. 2017. Genomic Disorders in Psychiatry-

387 What Does the Clinician Need to Know?. Current Psychiatry Reports. DOI: 10.1007/s11920-

$388 \quad 017-0831-5$.

389 Matson J.L., Shoemaker M. 2009. Intellectual disability and its relationship to autism spectrum

390 disorders. Research in Developmental Disabilities. DOI: 10.1016/j.ridd.2009.06.003.

391 Merikangas A.K., Segurado R., Heron E.A., Anney R.J.L., Paterson A.D., Cook E.H., Pinto D.,

392 Scherer S.W., Szatmari P., Gill M., Corvin A.P., Gallagher L. 2015. The phenotypic 
393 manifestations of rare genic CNVs in autism spectrum disorder. Molecular Psychiatry. DOI:

$39410.1038 / \mathrm{mp} .2014 .150$.

395 Miller D.T., Adam M.P., Aradhya S., Biesecker L.G., Brothman A.R., Carter N.P., Church D.M.,

396 Crolla J.A., Eichler E.E., Epstein C.J., Faucett W.A., Feuk L., Friedman J.M., Hamosh A.,

397 Jackson L., Kaminsky E.B., Kok K., Krantz I.D., Kuhn R.M., Lee C., Ostell J.M., Rosenberg C.,

398 Scherer S.W., Spinner N.B., Stavropoulos D.J., Tepperberg J.H., Thorland E.C., Vermeesch J.R.,

399 Waggoner D.J., Watson M.S., Martin C.L., Ledbetter D.H. 2010. Consensus statement:

400 Chromosomal microarray is a first-tier clinical diagnostic test for individuals with developmental

401 disabilities or congenital anomalies. American Journal of Human Genetics. DOI:

402 10.1016/j.ajhg.2010.04.006.

403 Miles J.H. 2011. Autism spectrum disorders-A genetics review. Genetics in Medicine. DOI:

404 10.1097/GIM.0b013e3181ff67ba.

405 Mitra I., Tsang K., Ladd-Acosta C., Croen L.A., Aldinger K.A., Hendren R.L., Traglia M.,

406 Lavillaureix A., Zaitlen N., Oldham M.C., Levitt P., Nelson S., Amaral D.G., Herz-Picciotto I.,

407 Fallin M.D., Weiss L.A., Flint J. 2016. Pleiotropic Mechanisms Indicated for Sex Differences in

408 Autism. PLOS Genetics DOI: 10.1371/journal.pgen.1006425.

409 Mulle J.G., Pulver A.E., McGrath J.A., Wolyniec P.S., Dodd A.F., Cutler D.J., Sebat J.,

410 Malhotra D., Nestadt G., Conrad D.F., Hurles M., Barnes C.P., Ikeda M., Iwata N., Levinson 
411 D.F., Gejman P.V., Sanders A.R., Duan J., Mitchell A.A., Peter I., Sklar P., O’Dushlaine C.T.,

412 Grozeva D., O’Donovan M.C., Owen M.J., Hultman C.M., Kähler A.K., Sullivan P.F., Kirov G.,

413 Warren S.T. 2014. Reciprocal Duplication of the Williams-Beuren Syndrome Deletion on

414 Chromosome 7q11.23 Is Associated with Schizophrenia. Biological Psychiatry. DOI:

415 10.1016/j.biopsych.2013.05.040.

416 Olson H., Shen Y., Avallone J., Sheidley B.R., Pinsky R., Bergin A.M., Berry G.T., Duffy F.H.,

417 Eksioglu Y., Harris D.J., Hisama F.M., Ho E., Irons M., Jacobsen C.M., James P., Kothare S.,

418 Khwaja O., Lipton J., Loddenkemper T., Markowitz J., Maski K., Megerian J.T., Neilan E.,

419 Raffalli P.C., Robbins M., Roberts A., Roe E., Rollins C., Sahin M., Sarco D., Schonwald A.,

420 Smith S.E., Soul J., Stoler J.M., Takeoka M., Tan W.H., Torres A.R., Tsai P., Urion D.K.,

421 Weissman L., Wolff R., Wu B.L., Miller D.T., Poduri A. 2014. Copy number variation plays an

422 important role in clinical epilepsy. Annals of Neurology. DOI: 10.1002/ana.24178.

423 Peycheva V., Kamenarova K., Ivanova N., Stamatov D., Avdjieva-Tzavella D., Alexandrova I.,

424 Zhelyazkova S., Pacheva I., Dimova P., Ivanov I., Litvinenko I., Bozhinova V., Tournev I.,

425 Simeonov E., Mitev V., Jordanova A., Kaneva R. 2018. Chromosomal microarray analysis of

426 Bulgarian patients with epilepsy and intellectual disability. Gene. DOI:

$427 \quad 10.1016 /$ j.gene.2018.05.015. 
428 Qiao Y., Riendeau N., Koochek M., Liu X., Harvard C., Hildebrand M.J., Holden J.J., Rajcan-

429 Separovic E., Lewis M.E. 2009. Phenomic determinants of genomic variation in autism spectrum

430 disorders. J Med Genet. DOI: 10.1136/jmg.2009.066795.

431 Schaefer G. 2016. Clinical Genetic Aspects of Autism Spectrum Disorders. International Journal

432 of Molecular Sciences. DOI: 10.3390/ijms17020180.

433 Schaefer G.B., Mendelsohn N.J. 2013. Clinical genetics evaluation in identifying the etiology of

434 autism spectrum disorders: 2013 guideline revisions. Genetics in Medicine. DOI:

$435 \quad 10.1038 /$ gim.2013.32.

436 Shaw-Smith C., Redon R., Rickman L., Rio M., Willatt L., Fiegler H., Firth H., Sanlaville D.,

437 Winter R., Colleaux L., Bobrow M., Carter N.P. 2004. Microarray based comparative genomic

438 hybridisation (array-CGH) detects submicroscopic chromosomal deletions and duplications in

439 patients with learning disability/mental retardation and dysmorphic features. J Med Genet. DOI:

$440 \quad 10.1136 / j m g .2003 .017731$.

441 Sokol D.K., Maloney B., Long J.M., Ray B., Lahiri D.K. 2011. Autism, Alzheimer disease, and

442 fragile X: APP, FMRP, and mGluR5 are molecular links. Neurology. DOI:

443 10.1212/WNL.0b013e3182166dc7. 
444 Stessman H.A.F., Xiong B., Coe B.P., Wang T., Hoekzema K., Fenckova M., Kvarnung M.,

445 Gerdts J., Trinh S., Cosemans N., Vives L., Lin J., Turner T.N., Santen G., Ruivenkamp C.,

446 Kriek M., van Haeringen A., Aten E., Friend K., Liebelt J., Barnett C., Haan E., Shaw M., Gecz

447 J., Anderlid B.M., Nordgren A., Lindstrand A., Schwartz C., Kooy R.F., Vandeweyer G.,

448 Helsmoortel C., Romano C., Alberti A., Vinci M., Avola E., Giusto S., Courchesne E., Pramparo

449 T., Pierce K., Nalabolu S., Amaral D.G., Scheffer I.E., Delatycki M.B., Lockhart P.J.,

450 Hormozdiari F., Harich B., Castells-Nobau A., Xia K., Peeters H., Nordenskjöld M., Schenck A.,

451 Bernier R.A., Eichler E.E. 2017. Targeted sequencing identifies 91 neurodevelopmental-disorder

452 risk genes with autism and developmental-disability biases. Nature Genetics. DOI:

$453 \quad 10.1038 /$ ng. 3792.

454 Tonnsen B.L., Boan A.D., Bradley C.C., Charles J., Cohen A., Carpenter L.A. 2016. Prevalence

455 of Autism Spectrum Disorders Among Children With Intellectual Disability. American Journal

456 on Intellectual and Developmental Disabilities. DOI: 10.1352/1944-7558-121.6.487.

457 de la Torre-Ubieta L., Won H., Stein J.L., Geschwind D.H. 2016. Advancing the understanding

458 of autism disease mechanisms through genetics. Nature Medicine. DOI: 10.1038/nm.4071.

459 Viñas-Jornet M., Esteba-Castillo S., Baena N., Ribas-Vidal N., Ruiz A., Torrents-Rodas D.,

460 Gabau E., Vilella E., Martorell L., Armengol L., Novell R., Guitart M. 2018. High Incidence of 
461 Copy Number Variants in Adults with Intellectual Disability and Co-morbid Psychiatric

462 Disorders. Behav Genet. DOI: 10.1007/s10519-018-9902-6.

463 Viscidi E.W., Triche E.W., Pescosolido M.F., McLean R.L., Joseph R.M., Spence S.J., Morrow

464 E.M., Marsit C.J. 2013. Clinical Characteristics of Children with Autism Spectrum Disorder and 465 Co-Occurring Epilepsy. PLoS ONE. DOI: 10.1371/journal.pone.0067797.

466 Vrijenhoek T., Middelburg E.M., Monroe G.R., van Gassen K.L.I., Geenen J.W., Hövels A.M.,

467 Knoers N.V., van Amstel H.K.P., Frederix G.W.J. 2018. Whole-exome sequencing in intellectual

468 disability; cost before and after a diagnosis. European Journal of Human Genetics. DOI:

469 10.1038/s41431-018-0203-6.

470 Wolfe K., Strydom A., Morrogh D., Carter J., Cutajar P., Eyeoyibo M., Hassiotis A., McCarthy

471 J., Mukherjee R., Paschos D., Perumal N., Read S., Shankar R., Sharif S., Thirulokachandran S.,

472 Thygesen JH., Patch C., Ogilvie C., Flinter F., McQuillin A., Bass N. 2017. Chromosomal

473 microarray testing in adults with intellectual disability presenting with comorbid psychiatric

474 disorders. European Journal of Human Genetics. DOI: 10.1038/ejhg.2016.107.

475 Whittington J., Holland A. 2018. A review of psychiatric conceptions of mental and behavioural

476 disorders in Prader-Willi syndrome. Neuroscience \& Biobehavioral Reviews. DOI:

477 10.1016/j.neubiorev.2018.10.006. 
478 Xu M., Ji Y., Zhang T., Jiang X., Fan Y., Geng J., Li F. 2018. Clinical Application of

479 Chromosome Microarray Analysis in Han Chinese Children with Neurodevelopmental

480 Disorders. Neuroscience Bulletin. DOI: 10.1007/s12264-018-0238-2.

481 


\section{Figure 1}

Frequency of pathogenic CNVs in both groups (ID and ASD) together stratified according to the clinical features

*heart, urogenital and brain , ADHD - hyperactivity, CNVs - copy number variants

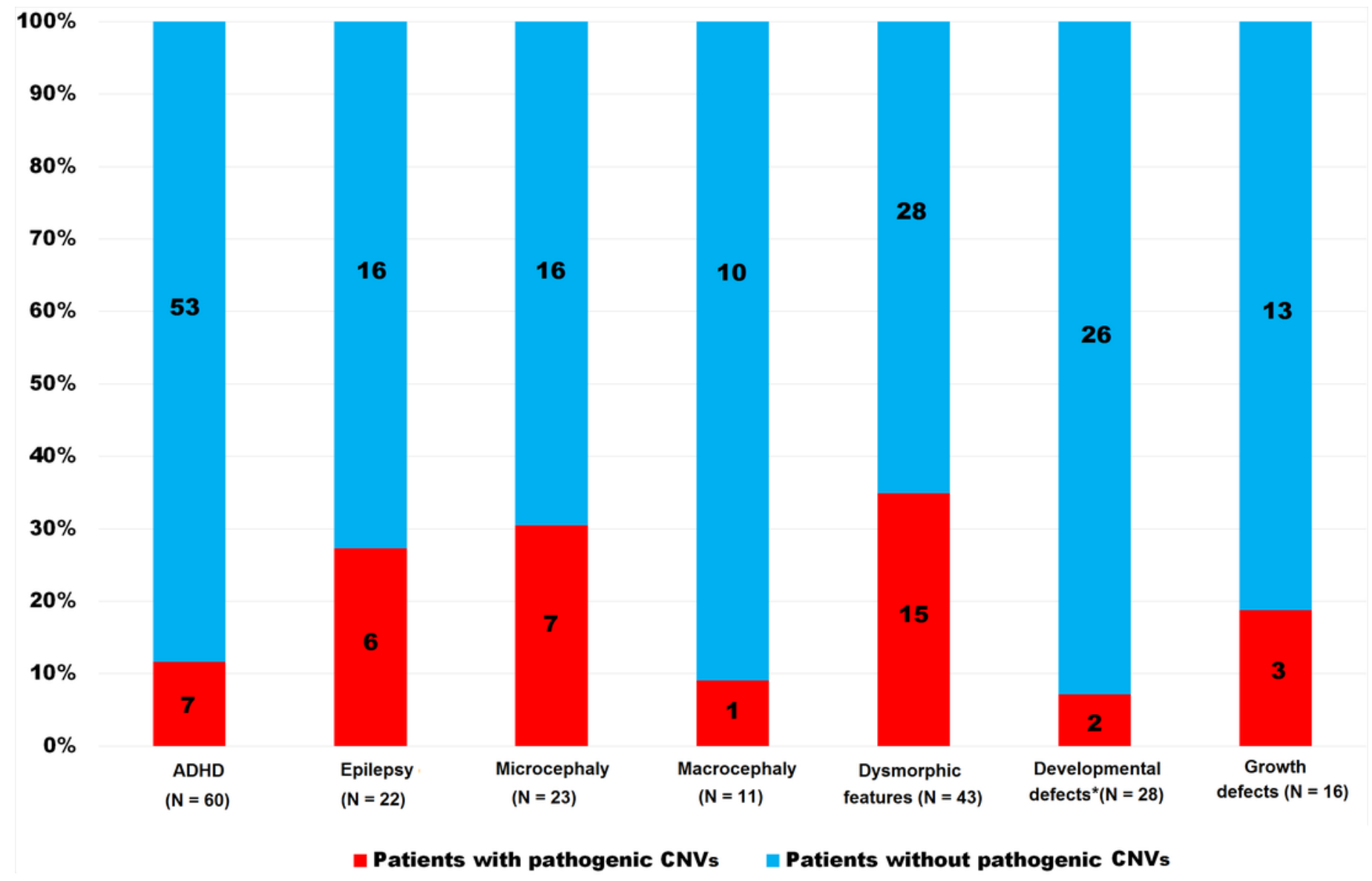


Figure 2

Frequency of pathogenic CNVs in ID group stratified according to the clinical features

*heart, urogenital and brain , ADHD - hyperactivity, CNVs - copy number variants
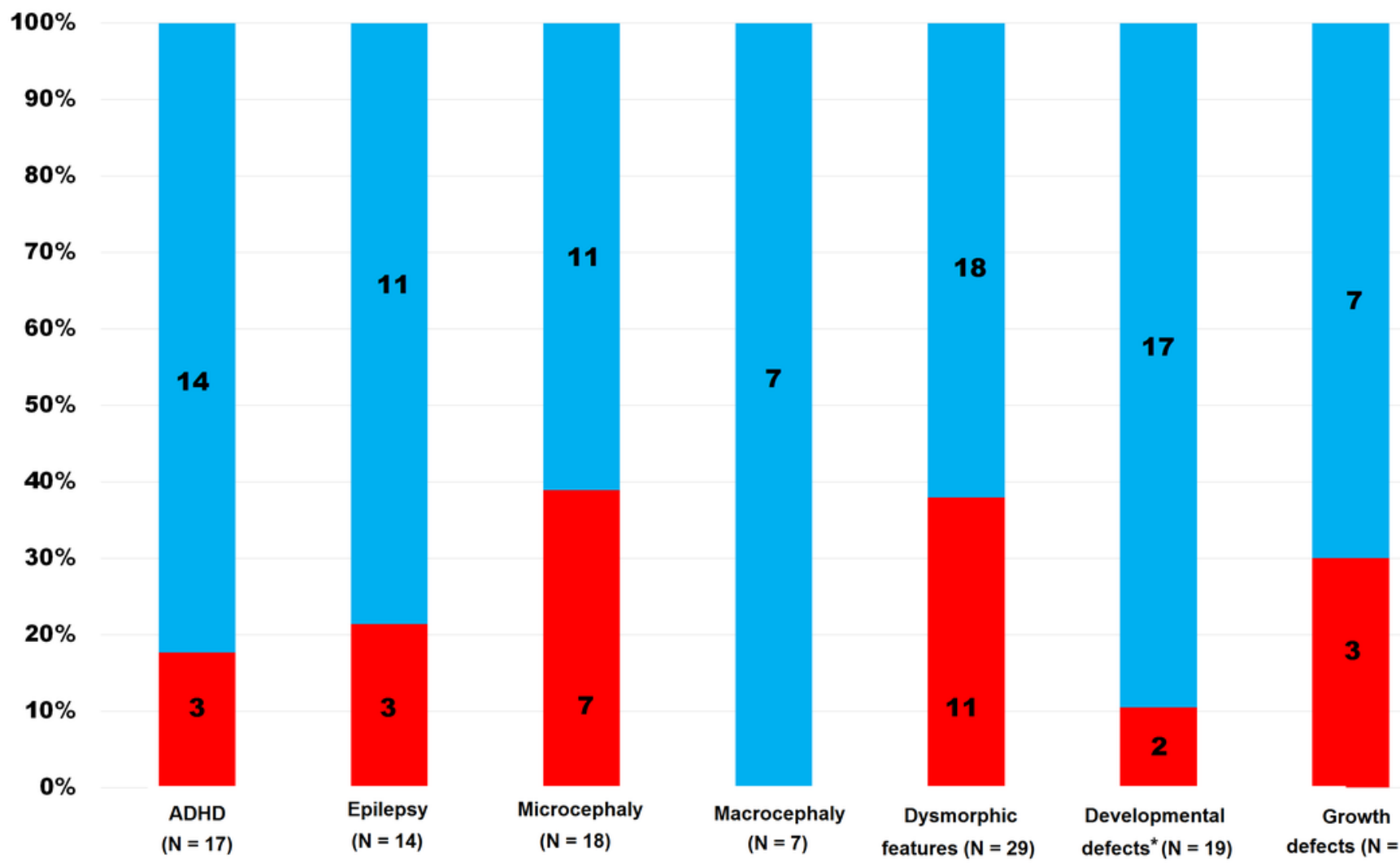

ADHD
$(\mathrm{N}=17)$

$(\mathrm{N}=14)$

Microcephaly
$(\mathrm{N}=18)$

Macrocephaly

Dysmorphic

Developmental

$(\mathrm{N}=7) \quad$ features $(\mathrm{N}=29)$

defects $^{\star}(\mathrm{N}=19)$ 
Figure 3

Frequency of pathogenic CNVs in ASD group stratified accordingto the clinical features

*heart, urogenital and brain , ADHD - hyperactivity, CNVs - copy number variants

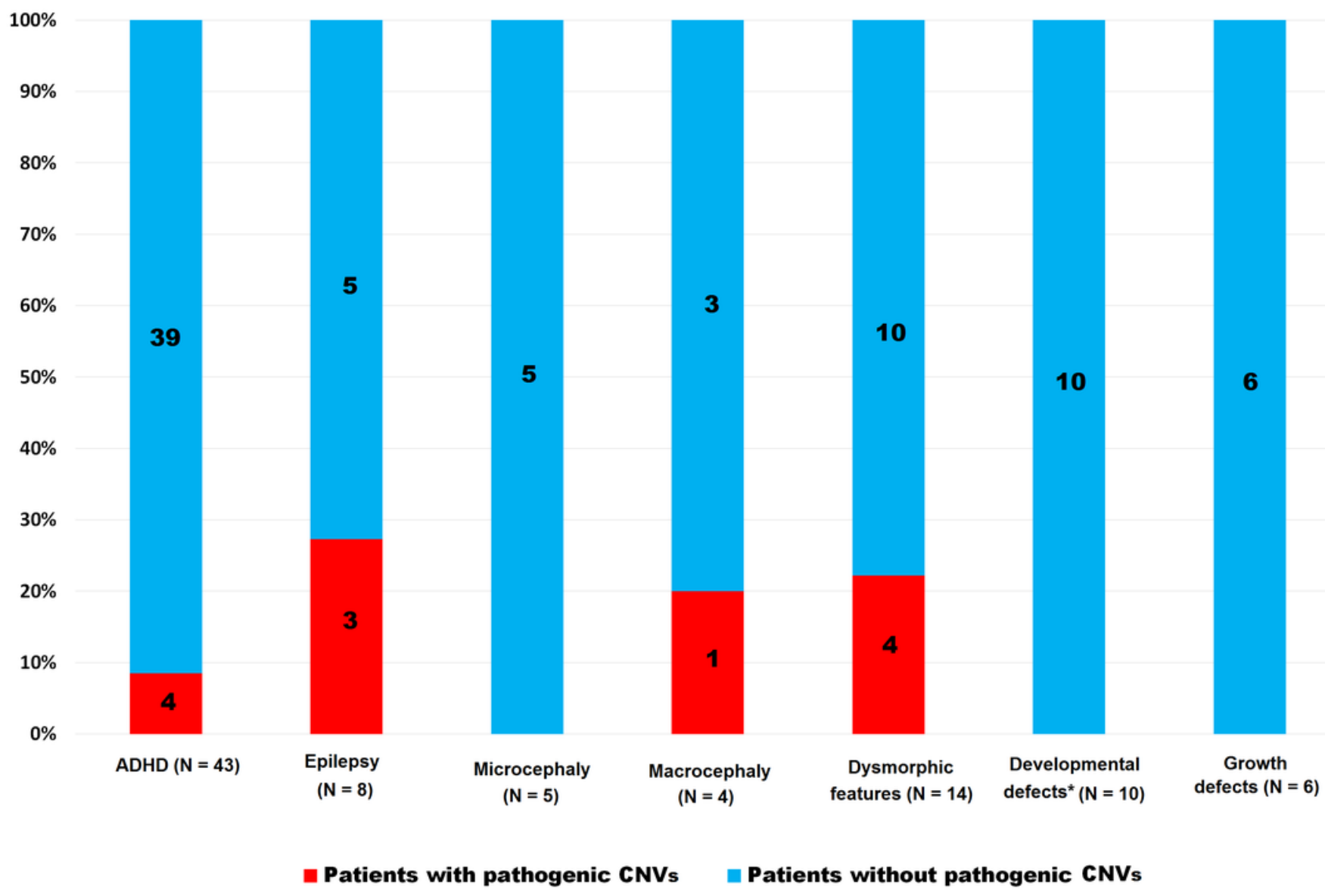




\section{Figure 4}

Differences between ID and ASD patients with epilepsy in pathogenic CNVspresentation

ID - intellectual disabilities patients, ASD - autism spectrum disorders patients, CNVs - copy number variants

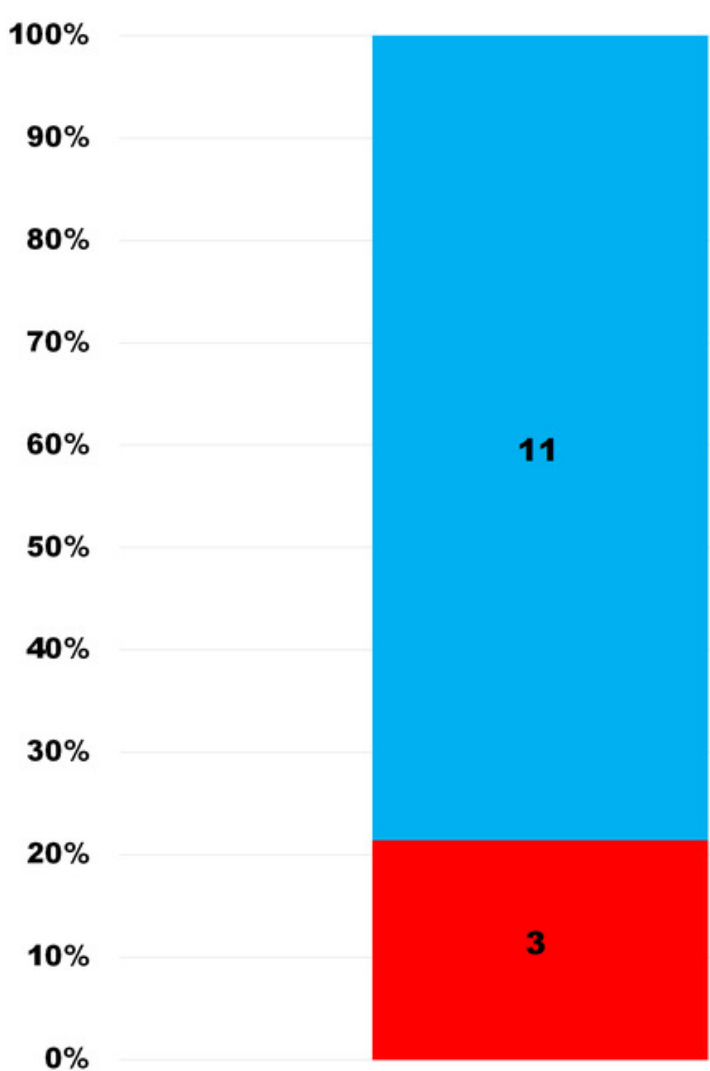

ID group

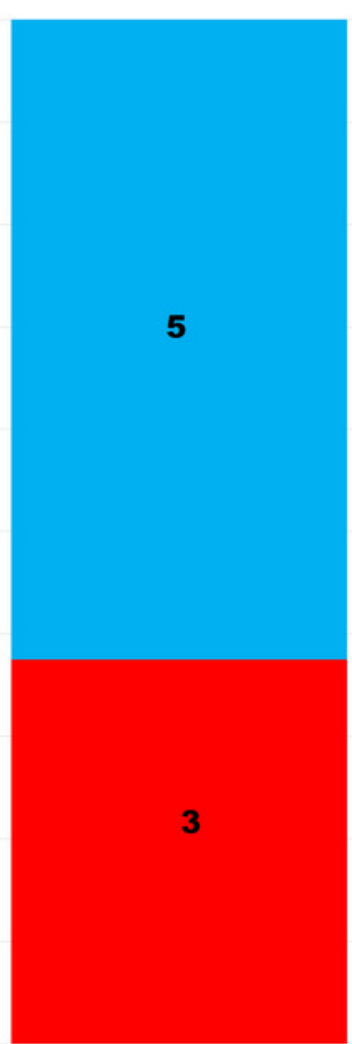

ASD group

a Patients with pathogenic CNVs a Patients without pathogenic CNVs 


\section{Figure 5}

Probability of pathogenic CNV in presence (A) /absence (B) of facial dysmorphia , epilepsy andmacrocephaly in ASD patients according to logistic regression

A - probability of pathogenic CNV in ASD patients with facial dysmorphia, epilepsy and macrocephaly, B probability of pathogenic CNV absence in ASD patients without facial dysmorphia, epilepsy and macrocephaly
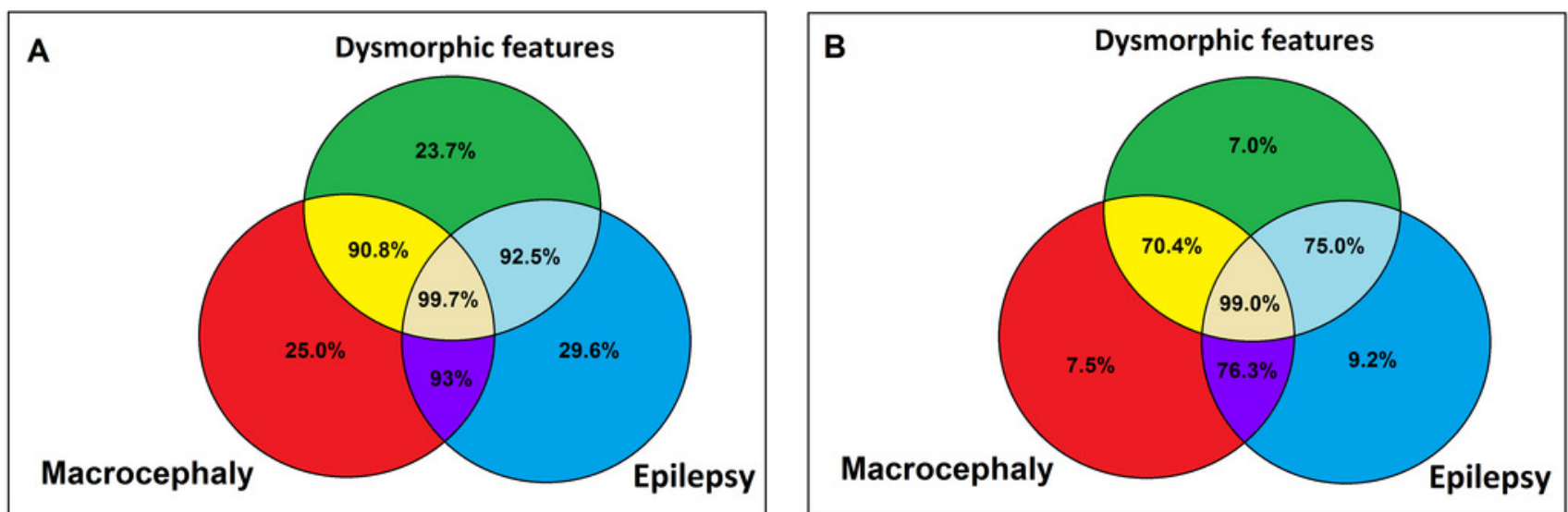
Figure 6

Formula for pathogenic CNVprobability calculation in ASD patients with epilepsy, macrocephaly anddysmorphism

$\beta$ estimation of parameter from logistic regression

$\mathrm{x}$ presence/absence of each clinical feature (epilepsy, macrocephaly, dysmorphism)

$$
P[Y(\boldsymbol{x})=1]=\frac{\exp \left(\boldsymbol{\beta}^{\prime} \boldsymbol{x}\right)}{1+\exp \left(\boldsymbol{\beta}^{\prime} \mathbf{x}\right)}
$$

$$
P[Y(\boldsymbol{x})=1]=\frac{\exp \left(\beta_{0}+\beta_{1} x_{1}+\beta_{2} x_{2}+\beta_{3} x_{3}\right)}{1+\exp \left(\beta_{0}+\beta_{1} x_{1}+\beta_{2} x_{2}+\beta_{3} x_{3}\right)}
$$




\section{Table $\mathbf{1}$ (on next page)}

Number of ID, ASD patients with each clinical features

*heart, urogenital and brain, ADHD - hyperactivity, ID - intellectual disabilities patients, ASD - autism spectrum disorders patients 


\begin{tabular}{lccc}
\multicolumn{1}{c}{ Clinical features } & $\begin{array}{c}\text { Number of } \\
\text { patients in ID } \\
\text { group (N = 90) }\end{array}$ & $\begin{array}{c}\text { Number of } \\
\text { patients in ASD } \\
\text { group (N = 114) }\end{array}$ & p-value \\
\hline ADHD & $17(18.9 \%)$ & $43(37.7 \%)$ & $p=0.003$ \\
Epilepsy & $14(15.6 \%)$ & $8(7.0 \%)$ & $\mathrm{ns}$ \\
Microcephaly & $18(20.0 \%)$ & $5(4.4 \%)$ & $p=0.001$ \\
Macrocephaly & $7(7.8 \%)$ & $4(3.5 \%)$ & $\mathrm{ns}$ \\
Dysmorphic features & $29(32.0 \%)$ & $14(12.3 \%)$ & $p=0.001$ \\
Developmental defects* & $18(20.0 \%)$ & $10(8.8 \%)$ & $p=0.025$ \\
Growth defects & $10(11.1 \%)$ & $6(5.3 \%)$ & $\mathrm{ns}$
\end{tabular}

1

2 


\section{Table 2 (on next page)}

Number ofpathogenic CNVs in ID and ASD patients with each clinical feature $S$

*heart, urogenital and brain , ADHD - hyperactivity, ID - intellectual disabilities patients, ASD - autism spectrum disorders patients, CNVs - copy number variants 


\begin{tabular}{lccc} 
Clinical features & $\begin{array}{c}\text { Pathogenic CNVs } \\
\text { in ID group } \\
\mathbf{N = 9 0}\end{array}$ & $\begin{array}{c}\text { Pathogenic CNVs in } \\
\text { ASD group } \\
\mathbf{N = 1 1 4}\end{array}$ & $\begin{array}{c}\text { Pathogenic CNVs } \\
\text { in both groups }\end{array}$ \\
\hline ADHD $(\mathrm{N}=60)$ & $17.65 \%(3 / 17)$ & $9.30 \%(4 / 43)$ & $11.67 \%(7 / 60)$ \\
Epilepsy $(\mathrm{N}=22)$ & $21.43 \%(3 / 14)$ & $37.50 \%(3 / 8)$ & $27.27 \%(6 / 22)$ \\
Microcephaly $(\mathrm{N}=23)$ & $38.89 \%(7 / 18)$ & $0.00 \%(0 / 5)$ & $30.43 \%(7 / 23)$ \\
Macrocephaly $(\mathrm{N}=11)$ & $0.00 \%(0 / 7)$ & $0.25 \%(1 / 4)$ & $9.09 \%(1 / 11)$ \\
Dysmorphic features $(\mathrm{N}=43)$ & $37.93 \%(11 / 29)$ & $28.57 \%(4 / 14)$ & $34.88 \%(15 / 43)$ \\
Developmental defects* $(\mathrm{N}=28)$ & $11.11 \%(2 / 18)$ & $0.00 \%(0 / 10)$ & $7.14 \%(2 / 28)$ \\
Growth defects $(\mathrm{N}=16)$ & $30.00 \%(3 / 10)$ & $0.00 \%(0 / 6)$ & $18.75 \%(3 / 16)$
\end{tabular}




\section{Table 3(on next page)}

Achieved significance of particular comorbidities topathogenic CNV finding in ASD and ID group by Fisher's exact test

*heart, urogenital and brain , ADHD - hyperactivity, ID - intellectual disabilities patients, ASD - autism spectrum disorders patients 


\begin{tabular}{lcc}
\multicolumn{1}{c}{ Clinical features } & $\boldsymbol{p}$-value in ID group & $\boldsymbol{p}$-value in ASD group \\
\hline ADHD & $\mathrm{ns}$ & $\mathrm{ns}$ \\
Epilepsy & $\mathrm{ns}$ & 0.01 \\
Microcephaly & 0.01 & $\mathrm{~ns}$ \\
Macrocephaly & $\mathrm{ns}$ & $\mathrm{ns}$ \\
Dysmorphic features & 0.05 & 0.01 \\
Developmental defects* & $\mathrm{ns}$ & $\mathrm{ns}$ \\
Growth defects & $\mathrm{ns}$ & $\mathrm{ns}$
\end{tabular}

